\title{
Review of Chlamydia; Are we testing the right people?
}

C Cunningham ${ }^{1}$, S Allstaff ${ }^{1}, \mathrm{R}_{\text {McGowan }}^{2}$, P McIntyre ${ }^{1}$

Tayside

${ }^{1}$ Ninewells Hospital and Medical school, ${ }^{2}$ Directorate of Public Health, NHS Tayside

\section{Background}

Locally we have observed an increase in Chlamydia (CT) testing in those over 25 years with a reduction in CT diagnoses. It is known that this age group carry less of a disease burden and as such we wished to explore whether testing was appropriate.

\section{Aims}

A study was designed to understand testing practices and the clinical indication for CT testing in this age group.

\section{Methods}

Laboratory CT test requests were configured to launch a series of pop-up boxes prompting the clinician to enter patient specific details. The study was piloted in the sexual health service for 2 weeks before being rolled out to all users. Data were collected for 3 months and included age, sex, test sample site, indication for testing/clinical details and result.
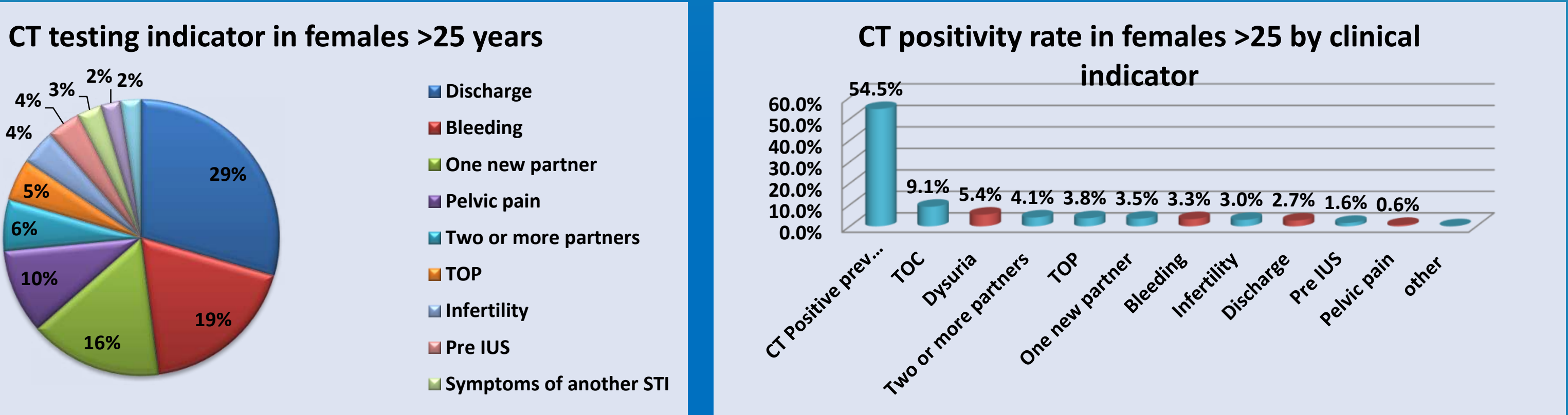

\section{CT testing indication in males $>25$ years}

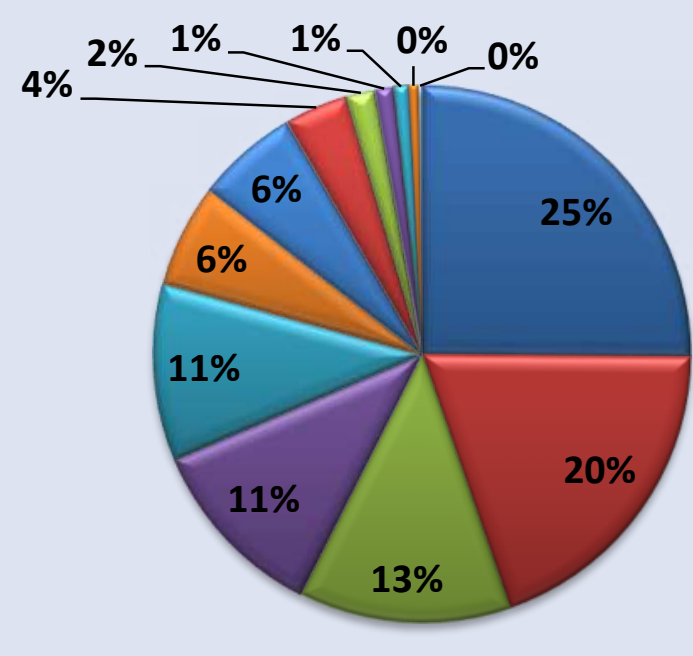

$\square$ One new partner
$\square$ Two or more partners
$\square$ Dysuria
$\square$ Testicular pain
$\square$ MSM
$\square$ Symptoms another STI
$\square$ Discharge
$\square$ Contact of CT
$\square$ TOC

\section{CT positivity rate in males $>25$ by clinical indicator}
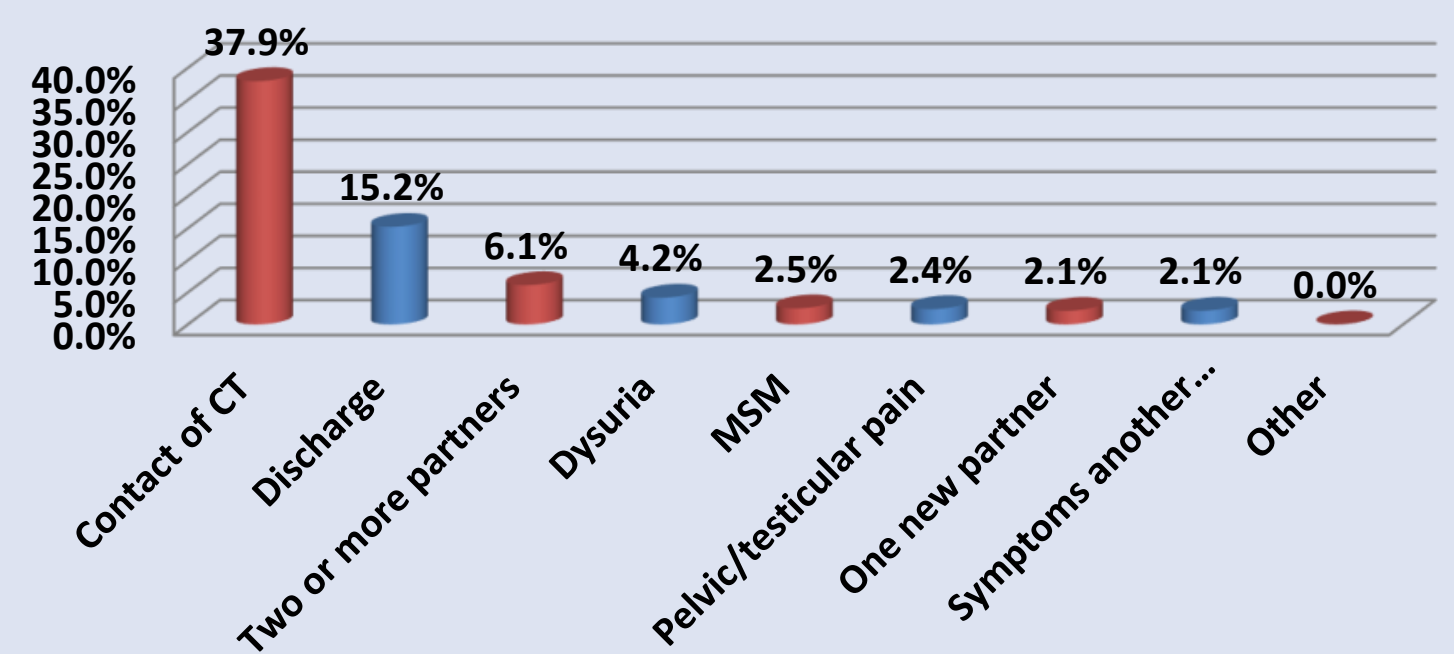

\section{Results}

From 2014 to 2017 the total number of CT tests and tests in women $\geq 25$ has remained static; in contrast the number of tests in men $\geq 25$ has increased by $85 \%$. In a total of 2388 samples the CT positivity rate regardless of symptoms was higher in men $(5.4 \%)$ than women $(3.2 \%, p<0.01)$. In females $(n=1629)$ the most common reason for testing was vaginal discharge $(29 \%)$, but this had a very low positivity rate $(2.7 \%)$, while the clinical indicator with the highest positivity rate $(54.5 \%)$ was previous $\mathrm{CT}$ infection in past 12 months. The most common reason for testing in males $(n=759)$ was a new partner in the past year $(25 \%)$ and the clinical indicator with the highest positivity rate was contact of a person with CT $(37.9 \%)$.

\section{Conclusion}

In the era of realistic medicine ${ }^{1}$ we wish to ensure the best use of public resources and target CT testing where prevalence is known to be highest. One in five women with diagnosed and treated Chlamydia are estimated to become re-infected within 10 months after initial treatment ${ }^{2}$ and this evidence marries with the testing indicators with the highest positivity rates. In contrast it is clear from this study that CT testing in women over 25 years with vaginal discharge has a very low positivity rate. As such we plan to produce local recommendations for CT testing and will continue to use the laboratory CT request prompts to educate and highlight the clinical indicators with low positivity rates in order to reduce unnecessary testing and will review testing practices after 1 year.

\section{REFERENCES}

1. Calderwood C. REALISTIC MEDICINE: Chief Medical Officer for Scotland 's Annual report 2014/15.

2. Hosenfeld CB, Workowski KA, Berman S, Zaidi A, Dyson J, Mosure D et al. Repeat infection with Chlamydia and gonorrhoea among females: a systematic review of the literature. Sex 